\title{
Search for invisible decays of $\pi^{0}, \eta, \eta^{\prime}, K_{S}$ and $K_{L}$ : A probe of new physics and tests using the Bell-Steinberger relation
}

\author{
S.N. Gninenko \\ Institute for Nuclear Research, Moscow 117312, Russia
}

(Dated: August 28, 2018)

\begin{abstract}
In the standard model the rate of the $\pi^{0}, \eta, \eta^{\prime}, K_{S}, K_{L} \rightarrow \nu \bar{\nu}$ decays is predicted to be extremely small. Therefore, an observation of any of these mesons $\left(M^{0}\right)$ decaying into an invisible final state would unambiguously signal the presence of new physics. The Bell-Steinberger relation connects $\mathrm{CP}$ and CPT violation in the mass matrix to $\mathrm{CP}$ and CPT violation in all decay channels of neutral kaons. It is a powerful tool for testing CPT invariance in the $K^{0}-\bar{K}^{0}$ system, assuming that there are no significant undiscovered decay modes of either $K_{S}$ or $K_{L}$ which could contribute to the precision of the results. The $K_{S}, K_{L} \rightarrow$ invisible decays have never been tested and the question of how much these decays can influence the Bell-Steinberger analysis of the $K^{0}-\bar{K}^{0}$ system still remains open. In the present work we propose a new experiment to search for the $M^{0} \rightarrow$ invisible decays which aims at probing new physics and answering this question. The experiment utilizes high-energy hadronic beams from the CERN SPS and the charge-exchange reactions of pions or kaons on nucleons of an active target, e.g. $\pi^{-}\left(K^{-}\right)+p \rightarrow M^{0}+n$, as a source of the well-tagged $M^{0}$ s emitted in the forward direction with the beam energy. If the decay $M^{0} \rightarrow$ invisible exists, it could be observed by looking for an excess of events with a specific signature: the complete disappearance of the beam energy in the detector. This unique signal of $M^{0} \rightarrow$ invisible decays allows for searches of the decays $K_{S}, K_{L} \rightarrow$ invisible with a sensitivity in the branching ratio $\operatorname{Br}\left(K_{S}\left(K_{L}\right) \rightarrow\right.$ invisible $) \lesssim 10^{-8}\left(10^{-6}\right)$, and $\pi^{0}, \eta, \eta^{\prime} \rightarrow$ invisible decays with a sensitivity a few orders of magnitude beyond the present experimental limits. This experiment is complementary to the one recently proposed for the search for invisible decays of dark photons and fits well with the present kaon physics program at CERN.
\end{abstract}

PACS numbers: 14.80.-j, 12.60.-i, 13.20.Cz, 13.35.Hb

\section{INTRODUCTION}

Experimental studies of invisible decays, i.e. particle transitions to an experimentally unobservable final state, played an important role both in the development of the standard model (SM) and in testing its extensions [1]. It is worth remembering the precision measurements of the $Z \rightarrow$ invisible decay rate for the determination of the number of lepton families in the SM. In recent years, experiments on invisible particle decays have received considerable attention. Motivated by various models of physics beyond the SM, these experiments include searches for invisible decays of $\pi^{0}$ mesons at E949 [2], $\eta$ and $\eta^{\prime}$ mesons at BES [3] , heavy $B$-meson decays at Belle [4], BaBAR [5], and BES [6], and invisible decays of the upsilon(1S) resonance at CLEO 7], baryonic number violation with nucleon disappearance at SNO 8], BOREXINO [9], and KamLAND [10], see also Ref. 11], electriccharge-nonconserving electron decays $e^{-} \rightarrow$ invisible [12], neutron-mirror-neutron oscillations at PSI [13] and the ILL reactor [14], and the disappearance of neutrons into another brane world [15]. One could also mention experiments looking for extra dimensions with invisible decays of positronium [16, 17], and proposals for new experiments to search for muonium annihilation into two neutrinos, $\mu^{+} e^{-} \rightarrow \nu \bar{\nu}$ [18], electric charge nonconservation in the muon decay $\mu^{+} \rightarrow$ invisible [19], and mirror-type dark matter through the invisible decays of orthopositronium in vacuum [20].
The use of the (pseudo)scalar mesons $\left(M^{0}\right)$, such as $\pi^{0}, \eta, \eta^{\prime}, K_{S}$, and $K_{L}$, to search for new physics by looking for their decays into invisible final states is advantageous, because in the standard model the rate of the $\pi^{0}, \eta, \eta^{\prime}, K_{S}, K_{L} \rightarrow \nu \bar{\nu}$ decays is predicted to be extremely small. For massless neutrino the decay $M^{0} \rightarrow \nu \bar{\nu}$ is forbidden kinematically by angular momentum conservation. Indeed, in the $M^{0}$ rest frame the neutrinos produced in the decay fly away in opposite directions along the same line. Since the neutrinos and antineutrinos are massless, the projection of the sum of their spins on this line equals \pm 1 . The projections of the orbital angular momentum of the neutrino on this line are equal to zero. Since in the initial state we have a scalar, the process is forbidden. For the case of massive neutrinos their spins in the rest frame must be opposite and, hence, one of the them is forced to have the "wrong" helicity. This results in the $M^{0} \rightarrow \nu \bar{\nu}$ decay rate being proportional to the neutrino mass squared:

$$
\Gamma\left(M^{0} \rightarrow \nu \bar{\nu}\right) \sim\left(\frac{m_{\nu}}{m_{M^{0}}}\right)^{2} \lesssim 10^{-16}
$$

Thus, we see that, if the decay $M^{0} \rightarrow$ invisible is observed it would unambiguously signal the presence of new physics, which could be due to, e.g. the existence of a new gauge boson with nonuniversal couplings to quarks, or the $M^{0}$ transitions into a hidden sector, or other effects [40].

Another motivation for searching for (in particular) the $K_{S}, K_{L} \rightarrow$ invisible decays is related to additional 
tests of the $K^{0}-\bar{K}^{0}$ system using the Bell-Steinberger relation 21]. This relation, obtained by using the unitarity condition, connects $\mathrm{CP}$ and $\mathrm{CPT}$ violation in the mass matrix of the kaon system, i.e. parameters describing $\mathrm{T}$ and $\mathrm{CPT}$ noninvariance, to $\mathrm{CP}$ and $\mathrm{CPT}$ violation in all decay channels of neutral kaons, see, e.g. Refs. [22 26]. We know that only CPT appears to be an exact symmetry of nature, while $\mathrm{C}, \mathrm{P}$ and $\mathrm{T}$ are known to be violated. Hence, testing the validity of CPT invariance probes the basis of the standard model. The Bell-Steinberger relation remains the most sensitive test of CPT symmetry. For example, the analyses of the KLOE Collaboration have reached the impressive sensitivity of $-5.3 \times 10^{-19}$ $\mathrm{GeV}<m_{K^{0}}-m_{\bar{K}^{0}}<6.3 \times 10^{-19} \mathrm{GeV}$ at $95 \%$ C.L. for the neural kaon mass difference [27]; see also Ref. 28].

Briefly, within the Wigner-Weisskopf approximation, the time evolution of the neutral kaon system is described by [27]:

$$
i \frac{d \Phi(t)}{d t}=H \Phi(t)=\left(M-i \frac{i}{c} \Gamma\right) \Phi(t)
$$

where $M$ and $\Gamma$ are $2 \times 2$ Hermitian matrices, which are time independent, and $\Phi(t)$ is a two-component state vector in the $K^{0}-\bar{K}^{0}$ space. Denoting by $m_{i j}$ and $\Gamma_{i j}$ the elements of $M$ and $\Gamma$ in the $K^{0}-\bar{K}^{0}$ basis, $C P T$ invariance implies

$$
\begin{array}{r}
m_{11}=m_{22} \quad\left(\text { or } \mathrm{m}_{\mathrm{K}^{0}}=\mathrm{m}_{\overline{\mathrm{K}}^{0}}\right) \text { and } \\
\Gamma_{11}=\Gamma_{22} \quad\left(\text { or } \Gamma_{\mathrm{K}^{0}}=\Gamma_{\overline{\mathrm{K}}^{0}}\right)
\end{array}
$$

The eigenstates of Eq. (2) can be written as

$$
\begin{array}{r}
K_{S, L}=\frac{1}{\sqrt{2\left(1+\left|\epsilon_{S, L}\right|^{2}\right)}}\left(\left(1+\epsilon_{S, L}\right) K^{0}\right. \\
\left. \pm\left(1-\epsilon_{S, L}\right) \bar{K}^{0}\right)
\end{array}
$$

with

$$
\begin{gathered}
\epsilon_{S, L}=\frac{1}{m_{L}-m_{S}+i\left(\Gamma_{S}-\Gamma_{L}\right) / 2}\left[-i \operatorname{Im}\left(\mathrm{m}_{12}\right)-\right. \\
\frac{1}{2} \operatorname{Im}\left(\Gamma_{12}\right) \pm \frac{1}{2}\left(\mathrm{~m}_{\overline{\mathrm{K}}^{0}}-\mathrm{m}_{\mathrm{K}^{0}}-\frac{\mathrm{i}}{2}\left(\Gamma_{\overline{\mathrm{K}}^{0}}-\Gamma_{\mathrm{K}^{0}}\right)\right] \equiv \epsilon \pm \delta
\end{gathered}
$$

The unitarity condition allows us to express the four elements of $\Gamma$ in terms of appropriate combinations of the kaon decay amplitudes $A_{i}$ :

$$
\Gamma_{i j}=\sum_{f} A_{i}(f) A_{j}^{*}(f), \quad i, j=1,2=K^{0}, \bar{K}^{0}
$$

where the sum is over all the accessible final states.

$$
\begin{array}{r}
\left(\frac{\Gamma_{S}+\Gamma_{L}}{\Gamma_{S}-\Gamma_{L}}+i \tan \phi_{\mathrm{SW}}\right)\left(\frac{\operatorname{Re}(\epsilon)}{1+|\epsilon|^{2}}-\mathrm{i} \operatorname{Im}(\delta)\right) \\
=\frac{1}{\Gamma_{S}-\Gamma_{L}} \sum_{F} A_{L}(f) A_{S}^{*}(f),
\end{array}
$$

where $\phi_{S W}=\arctan \left[2\left(\mathrm{~m}_{\mathrm{L}}-\mathrm{m}_{\mathrm{S}}\right) /\left(\Gamma_{\mathrm{S}}-\Gamma_{\mathrm{L}}\right)\right]$. One can see that the Bell-Steinberger relation (7) relates a possible violation of CPT invariance $\left(m_{K^{0}}=m_{\bar{K}^{0}}\right.$ and/or $\left.\Gamma_{K^{0}}=\Gamma_{\bar{K}^{0}}\right)$ in the $K^{0}-\bar{K}^{0}$ system to the observable CP-violating interference of $K_{S}$ and $K_{L}$ decays into the same final state $f$. If CPT invariance is not violated, then $\operatorname{Im}(\delta)=0$. We stress that any evidence for $\operatorname{Im}(\delta) \neq 0$ resulting from this relation can only manifest the violation of CPT or unitarity [23].

Generally, the advantage of the neutral kaon system is attributed to the fact, that only a few (hadronic) decay modes give significant contributions to the rhs of Eq. (77). In particular, it is assumed that there are no significant contributions from invisible decay modes of either $K_{L}$ or $K_{S}$ which, however, have never been experimentally tested. Therefore, the contribution from these decay modes and how much the errors on $\operatorname{Re}(\epsilon)$ and $\operatorname{Im}(\delta)$ would increase if the invisible modes have maximal $\mathrm{CP}$ violation are still open questions, see, e.g., Ref. [29]. As long as these questions are not answered experimentally, further tests of CPT symmetry via Bell-Steinberger relations remain important.

In order to estimate the contribution from the $K_{S}, K_{L} \rightarrow$ invisible decay to the right-hand side of Eq.(7) one can follow the same procedure used by the KLOE Collaboration for the estimation of the contribution from the $K_{L}, K_{S} \rightarrow \pi^{0} \pi^{0} \pi^{0}$ channel to Eq.(7) [27]. For invisible states we define

$$
\begin{array}{r}
\alpha_{i n v} \equiv \frac{1}{\Gamma_{S}}<A_{L}(i n v) A_{S}^{*}(i n v)>= \\
\frac{\tau_{K_{S}}}{\tau_{K_{L}}} \eta_{i n v}^{*} \operatorname{Br}\left(K_{L} \rightarrow \text { invisible }\right)
\end{array}
$$

where $\eta_{i n v}$ coefficient is the $A_{L}(i n v), A_{S}(i n v)$ amplitude ratio. As there is no experimental limit on $\eta_{i n v}^{*}$ it would be more convenient to set a bound on $\left|\alpha_{i n v}\right|$ by using the relation

$$
\left|\alpha_{i n v}\right|^{2}=\frac{\tau_{K_{S}}}{\tau_{K_{L}}} \operatorname{Br}\left(K_{L} \rightarrow \text { invisible }\right) \operatorname{Br}\left(K_{S} \rightarrow \text { invisible }\right)
$$

and experimental constraints on the rate of $K_{S}, K_{L} \rightarrow$ invisible decays (derived below). Equation (9) is based on the assumption that the $K_{L}\left(K_{S}\right) \rightarrow$ invisible decay mode is dominated by a single $C P$-conserving (violating) amplitude. Note that all quantum numbers of the invisible final state have to be equal between $K_{L}$ and $K_{S}$ decays in order to allow for interference between two amplitudes on the right-hand side of Eq.(17).

If the decays $K_{S}, K_{L} \rightarrow$ invisible exist they will contribute to the total $K_{L}, K_{S}$ decay rate

$$
\begin{array}{r}
\Gamma_{K_{L}\left(K_{S}\right)=} \sum_{i} \Gamma_{i}\left(K_{L}\left(K_{S}\right) \rightarrow \text { visible }\right) \\
+\Gamma\left(K_{L}\left(K_{S}\right) \rightarrow \text { invisible }\right)
\end{array}
$$

resulting in

$$
\sum_{i} B r_{i}\left(K_{L}\left(K_{S}\right) \rightarrow \text { visible }\right)<1
$$


In order to obtain bounds on the branching fraction $\operatorname{Br}\left(K_{L}\left(K_{S}\right) \rightarrow\right.$ invisible $)$, to estimate the allowed extra contribution of $K_{S}, K_{L} \rightarrow$ invisible decays to Eq. (10), and to derive a limit on $\left|\alpha_{i n v}\right|^{2}$, we use the results of the most precise measurements of the branching fractions of the visible $K_{S}, K_{L}$ decay modes from Particle Data Group PDG [1]. Summing up all measured branching fractions, we get

$$
\sum_{i} B r_{i}\left(K_{S} \rightarrow \text { visible }\right)=1.00068 \pm 0.00048
$$

and

$$
\sum_{i} B r_{i}\left(K_{L} \rightarrow \text { visible }\right)=1.00032 \pm 0.00058
$$

resulting, respectively, in

$$
\operatorname{Br}\left(K_{S} \rightarrow \text { invisible }\right)<1.1 \times 10^{-4},(95 \% \text { C.L. }),
$$

and

$$
\operatorname{Br}\left(K_{L} \rightarrow \text { invisible }\right)<6.3 \times 10^{-4},(95 \% \text { C.L. }) .
$$

Using for the $K_{S}$ and $K_{L}$ lifetime the averages $\tau_{K_{S}}=$ $0.08958 \pm 0.00006 \mathrm{~ns}$ and $\tau_{K_{L}}=51.16 \pm 0.2 \mathrm{~ns}$, we obtain

$$
\left|\alpha_{i n v}\right|<2.8 \times 10^{-5},(95 \% \text { C.L. }) .
$$

Note that the averages and errors for the branching fractions for the visible $K_{S}, K_{L}$ decay modes were obtained by PDG with a fit procedure. For comparison, we also try to estimate $\left|\alpha_{i n v}\right|$ by using results from direct measurements of $B r_{i}\left(K_{S}\left(K_{L}\right) \rightarrow\right.$ visible), obtained mostly by the KLOE and NuTeV Collaborations [1]. For the $K_{S}$ and $K_{L}$ lifetimes quoted above the new limit on $\left|\alpha_{i n v}\right|$ is similar to the one from Eq.(16) within a factor of 2 .

It is interesting to compare these results with the limit on the contribution of the $3 \pi^{0}$ decay modes to the BellSteinberger relation derived by the KLOE Collaboration, $\left|\alpha_{\pi^{0} \pi^{0} \pi^{0}}\right|<7 \times 10^{-6}$ at $95 \%$ C.L. [27]. It should be noted, that $K_{S}, K_{L}$ decay parameters from the decay channels with the branching ratio $\operatorname{Br}\left(K_{S} \rightarrow f\right)=$ $\Gamma\left(K_{S} \rightarrow f\right) / \Gamma_{S} \gtrsim 10^{-5}$ and $\operatorname{Br}\left(K_{L} \rightarrow f\right) \Gamma_{L} / \Gamma_{S} \gtrsim 10^{-5}$ are within the present accuracy of Eq.(7) and contribute to the Bell-Steinberger analysis of the kaon system [23]. Therefore, an improvement of the bounds of Eq. (14) and 15) by at least an order of magnitude is necessary in order to agree with the present accuracy of Eq.(7). The decay mode that gives the largest contribution to the precision of the Bell-Steinberger analysis at the level $O\left(10^{-5}\right)$ is now $K_{L} \rightarrow \pi^{+} \pi^{-}$through the uncertainty on the phase $\phi+-[27]$.

One of the aims of this work is to show that the limits of Eqs. (14)15) can be significantly improved by the proposed experiment that would search for the still unexplored decay modes $K_{S}, K_{L} \rightarrow$ invisible with a high-energy $K^{ \pm}$beam at the CERN SPS. The expected sensitivity in the branching fraction is $\operatorname{Br}\left(K_{S}\left(K_{L}\right) \rightarrow\right.$ invisible $) \lesssim 10^{-8}\left(10^{-6}\right)$. The experiment is also capable of a sensitive search for $\pi^{0}, \eta, \eta^{\prime} \rightarrow$ invisible decays and could improve the existing limits by more than an order of magnitude. The rest of the paper is organized as follows. The method of the search and the experimental setup are described in Sec. II, the background sources are discussed in Sec. III, and the expected sensitivity for the decay $M \rightarrow$ invisible is presented in Sec. IV. Section $\mathrm{V}$ contains concluding remarks.

\section{AN EXPERIMENT TO SEARCH FOR $\pi^{0}, \eta, \eta^{\prime}, K^{0} \rightarrow$ invisible DECAYS}

The detector specifically designed to search for the $\pi^{0}, \eta, \eta^{\prime}, K_{S}, K_{L} \rightarrow$ invisible decays is schematically shown in Fig. 1, This experimental setup is complementary to the one recently proposed for the search for invisible decays of dark photons at the SPS at CERN [31, 32]. The experiment could employ, e.g., the H4 hadron beam, which is produced in the target T2 of the SPS and transported to the detector in an evacuated beamline tuned to a freely adjustable beam momentum from $10-300 \mathrm{GeV} / \mathrm{c}$ 30]. The typical maximal beam intensity at $\simeq 50-100$ $\mathrm{GeV}$, is of the order of $\simeq 10^{7} \pi^{ \pm}$and $\simeq 10^{6} K^{ \pm}$for one SPS spill with $10^{12}$ protons on target. The typical SPS cycle for fixed-target (FT) operation lasts $14.8 \mathrm{~s}$, including $4.8 \mathrm{~s}$ spill duration. The maximal number of FT cycles is four per minute. The beam has high purity: the admixture of the other charged particles is below $10^{-2}$. The beam can be focused onto a spot of the order of a few $\mathrm{cm}^{2}$.

We first consider the experiment that would search for the $K_{S}, K_{L} \rightarrow$ invisible decays. The method of the search is as follows. The source of $K^{0}\left(\bar{K}^{0}\right) \mathrm{s}$ is the chargeexchange reaction of high-energy kaons on nucleons of an active target

$$
\begin{aligned}
& K^{-}+p \rightarrow \bar{K}^{0}+n, \text { or } \\
& K^{+}+n \rightarrow K^{0}+p
\end{aligned}
$$

where the neutral kaon is emitted mainly in the forward direction with the beam momentum and the recoil nucleon carries away a small fraction of the beam energy. Further, we will assume no difference between these two reactions. The invisible decay $K^{0} \rightarrow$ invisible is expected to be a very rare event which occurs with a rate much smaller than the total $K^{0}$ production rate. Hence, its observation presents a challenge for the design and performance of the detector.

The detector shown in Fig. 1 is equipped with the scintillating counters S1 and S2 (which define the beam), an active target $T$ surrounded by a high-efficiency electromagnetic calorimeter (ECAL) serving as a veto against photons and other secondaries emitted from the target at large angles, high-efficiency forward veto counters V1 and $\mathrm{V} 2$, a decay volume DV, and a massive, hermetic hadronic calorimeter (HCAL) located at the end of the 


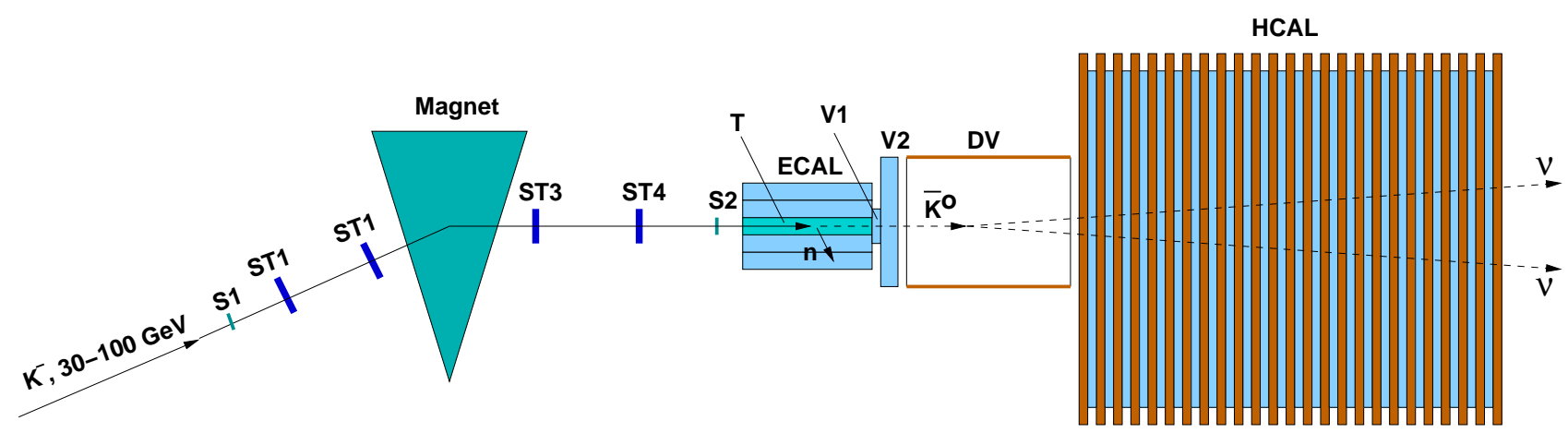

FIG. 1: Schematic illustration of the setup to search for the invisible decays of neutral kaons in the proposed experiment at high energies. The beam of incident charged kaons is defined by the scintillating counters $\mathrm{S}_{1,2}$. The momentum of the beam is additionally selected with a momentum spectrometer consisting of a dipole magnet and a low-density tracker, made of a set of straw tube chambers (ST1-ST3) or Micromegas detectors. The $K^{0}$ s are produced in the charge-exchange reaction of kaons scattering off nuclei in the active target $\mathrm{T}$. The $\mathrm{T}$ is surrounded by the Veto system consisting of the electromagnetic calorimeter (ECAL) and two high-efficiency scintillating counters V1 and V2 used against photons or charged secondaries that could escape the target at a large angle or in the forward direction, and a massive completely hermetic hadronic calorimeter (HCAL) to absorb the energy of all secondaries. The $K^{0}$ s either decay invisibly in the target, or they penetrates V1 and V2 without interactions and (as shown) decay invisibly in flight in the decay volume DV into, e.g., a pair of heavy $\nu$, which carry away almost all of the primary beam energy, resulting in the zero-energy signature in the detector. The recoil neutron shown typically deposits a small amount of energy. The same setup can be used for the searches for the $\pi^{0}, \eta, \eta^{\prime} \rightarrow$ invisible decays (see text.

setup to detect energy deposited by secondaries from the primary interactions $K^{ \pm} A \rightarrow$ anything of $K^{ \pm} \mathrm{S}$ with nuclei $A$ in the target. For searches at low energies, Cherenkov counters to enhance the incoming hadron tagging efficiency can be used.

The reaction (17) occurs practically uniformly over the length of the target. The fraction of the primary kaon (pion) energy deposited in the target is used to determine the position of the interaction vertex along the beam direction. The produced $K^{0}$ - composed of equal portions of $K_{S}$ and $K_{L^{-}}$either decay quickly in the target $T$, or penetrates the veto system without interactions and either decays in flight in the DV or interacts in the HCAL. If the $K_{S}$ and $K_{L}$ decay invisibly, it is assumed that the final-state particles in this case also penetrate the rest of the detector without prompt decay into ordinary particles, which could deposit energy in the HCAL. In order to suppress background due to the detection inefficiency, the detector must be longitudinally completely hermetic. To enhance detector hermeticity, the hadronic calorimeter has a total thickness of $\simeq 28 \lambda_{\text {int }}$ (nuclear interaction lengths).

The occurrence of $K_{S}, K_{L} \rightarrow$ invisible decays produced in $K^{ \pm}$interactions would appear as an excess of events with a signal in the $T$, see Fig. 1 and zero energy deposition in the rest of the detector (i.e. above that expected from the background sources). Thus, the signal candidate events have the signature

$$
S_{K^{0} \rightarrow \text { invisible }}=\mathrm{T} \cdot \overline{\mathrm{V} 1 \cdot \mathrm{V} 2 \cdot \mathrm{HCAL}}
$$

and should satisfy the following selection criteria.

(i) The measured momentum of the incoming kaon should correspond to its selected value. (ii) The kaon should enter the target and the interaction vertex should be localized within the target volume.

(iii) The should be no energy deposition in the ECAL veto, $\mathrm{V} 1$ and $\mathrm{V} 2$.

(iv) The fraction of the beam energy deposited in the HCAL modules should be consistent with zero.

The application of all the previous considerations to the search for the decays $\pi^{0}, \eta, \eta^{\prime} \rightarrow$ invisible with the same detector is straightforward. The source of $\pi^{0}, \eta, \eta^{\prime}$ is the charge-exchange reaction of high energy pions on nucleons of the target

$$
\begin{aligned}
& \pi^{-}+p \rightarrow \pi^{0}, \eta, \eta^{\prime}+n, \quad \text { or } \\
& \pi^{+}+n \rightarrow \pi^{0}, \eta, \eta^{\prime}+p,
\end{aligned}
$$

where the final-state neutral meson is emitted mainly in the forward direction with the beam momentum and the recoil nucleon carries away a small fraction of the beam energy. Similar to the $K$-meson case, the occurrence of $\pi^{0}, \eta, \eta^{\prime} \rightarrow$ invisible decays produced in $\pi^{ \pm}$interactions would appear as an excess of events with the same signature of Eq.(18), i.e. the presence of a signal in the $T$, see Fig. 1 and zero energy deposition in the rest of the detector (i.e. above that expected from the background sources).

In Fig 2 the expected distribution of the signal of Eq.(18) in the HCAL is shown, i.e. the pedestal sum over the HCAL modules, representing the signal from an invisible final state in units of hadronic energy. The FWHM of the signal peak is expected to be $\lesssim 100 \mathrm{MeV}$. The distribution was estimated from the real data taken 


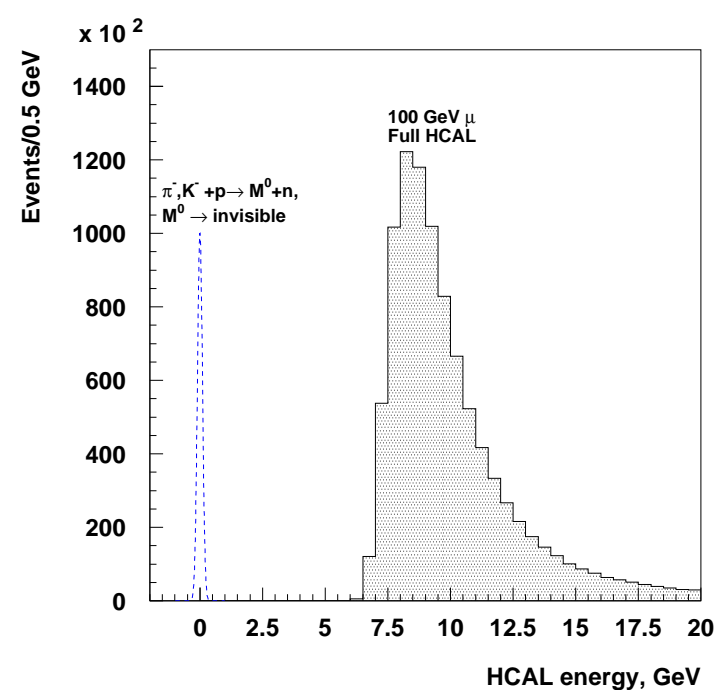

FIG. 2: The distribution of the energy deposited in the HCAL by traversing muons with energy $E_{\mu}=100 \mathrm{GeV}$. The peak of the pedestal sum over the HCAL is also shown, representing the signal from the invisible final state in units of hadronic energy. The FWHM of the signal peak is expected to be $\lesssim 100$ $\mathrm{MeV}$.

at the $\mathrm{H} 4$ beam. The simulated distribution of the energy deposited in the HCAL by traversing muons with energy $E_{\mu}=100 \mathrm{GeV}$ is also shown for comparison.

\section{BACKGROUND}

The background reactions resulting in the signature of the process $\pi, K+p \rightarrow M^{0}+n ; M^{0} \rightarrow$ invisible, see Eq. (18), can be classified as being due to physical- and beamrelated sources. To perform a full detector simulation in order to investigate these backgrounds down to the level $\lesssim 10^{-10}$ would require a prohibitively large amount of computer time. Consequently, only the following sources of background - identified as the most dangerous - are considered and evaluated with reasonable statistics combined with numerical calculations:

(i) One of the main background sources is related to the low-energy tail in the distribution of the energy of the primary hadronic beam. This tail is caused by the beam interactions with a passive material, such as the entrance windows of the beam lines, residual gas, etc. Another source of low-energy hadrons is due to their decays in flight in the beam line when the low energy decay pions or muons mimic the signature Eq. (18) in the detector. The uncertainties arising from the lack of knowledge of the dead material composition in the beam line are potentially the largest source of systematic uncertainty in accurate calculations of the fraction and energy distribution of these events. An estimation shows that the fraction of events with energy below $\lesssim 10 \mathrm{GeV}$ in the hadron beam tuned, e.g., to $50 \mathrm{GeV}$ could be as large as $10^{-8}-10^{-6}$. Hence, the sensitivity of the experiment could be determined by the presence of such particles in the beam, unless one takes special measures to suppress this background. To improve the primary high-energy hadron selection and suppress background from the possible admixture of low-energy particles, one can use a tagging system utilizing the magnetic spectrometer installed upstream of the detector, as schematically shown in Fig. [1.

(ii) The fake signature of Eq. (18) could also arise when the $K^{0}$ from the reaction (17) or a leading hadron, $h$, from the reaction $\pi+A \rightarrow h+\ldots$ that occurred in the target is not detected due to the incomplete hermeticity of the HCAL. In this case, the produced $K^{0}$ punches through the HCAL without depositing energy above a certain threshold $E_{t h}$. This effect is illustrated in Fig. 3(a), which shows the distribution of energy deposited by $K^{0}$ s produced at $95 \mathrm{GeV}$, in two consecutive HCAL modules $\left(\simeq 14 \lambda_{\text {int }}\right)$. The distribution is obtained with GEANT4 simulations [33]. The peak of events at zero energy in the spectrum is caused by the punch-through neutral kaons. Those events with a sum of energy released in two HCAL modules below the threshold $E_{t h} \simeq 0.1 \mathrm{GeV}$ are considered as zero-energy events. In Fig. 3(b), one can also see that a similar distribution of energy deposited by $K^{0} \mathrm{~s}$ in four consecutive HCAL modules $\left(\simeq 28 \lambda_{\text {int }}\right)$ has no such zero-energy events.

The punch-through probability is defined roughly by $\simeq \exp \left(-L_{H C A L} / \lambda_{\text {int }}\right)$, where $L_{H C A L}$ is the HCAL thickness. It is $\simeq 10^{-12}$ for the total thickness of the HCAL about $28 \lambda_{\text {int }}$. Since performing detector simulations at this level of precision is not possible, the rough estimate of the HCAL nonhermeticity for high-energy hadrons was cross-checked with GEANT4-based simulations in the following way. The low-energy tail in the distribution of energy deposited in the full HCAL by $\simeq 10^{7}$ simulated neutral kaons was fitted by a smooth polynomial function and extrapolated to the low-energy region in order to evaluate the number of events below a certain threshold $E_{t h}$, see Ref. [32] for more details. This procedure results in an estimate of the HCAL nonhermeticity, defined as the ratio of the number of events below the threshold $E_{t h}$ to the total number of incoming particles, $\left(E<E_{t h}\right) / n_{t o t}$. For example, for the energy threshold $E_{t h} \simeq 0.5 \mathrm{GeV}$ the nonhermeticity is found to be at the level $\simeq 0.4 \times 10^{-11}$, which is in satisfactory agreement with the above estimate when taking into account the accuracy of this procedure. This results in an overall conservative level for this background of $\lesssim 10^{-13}$ per incident beam kaon reaction in the target.

(iii) Another type of process which could mimic the tag- 

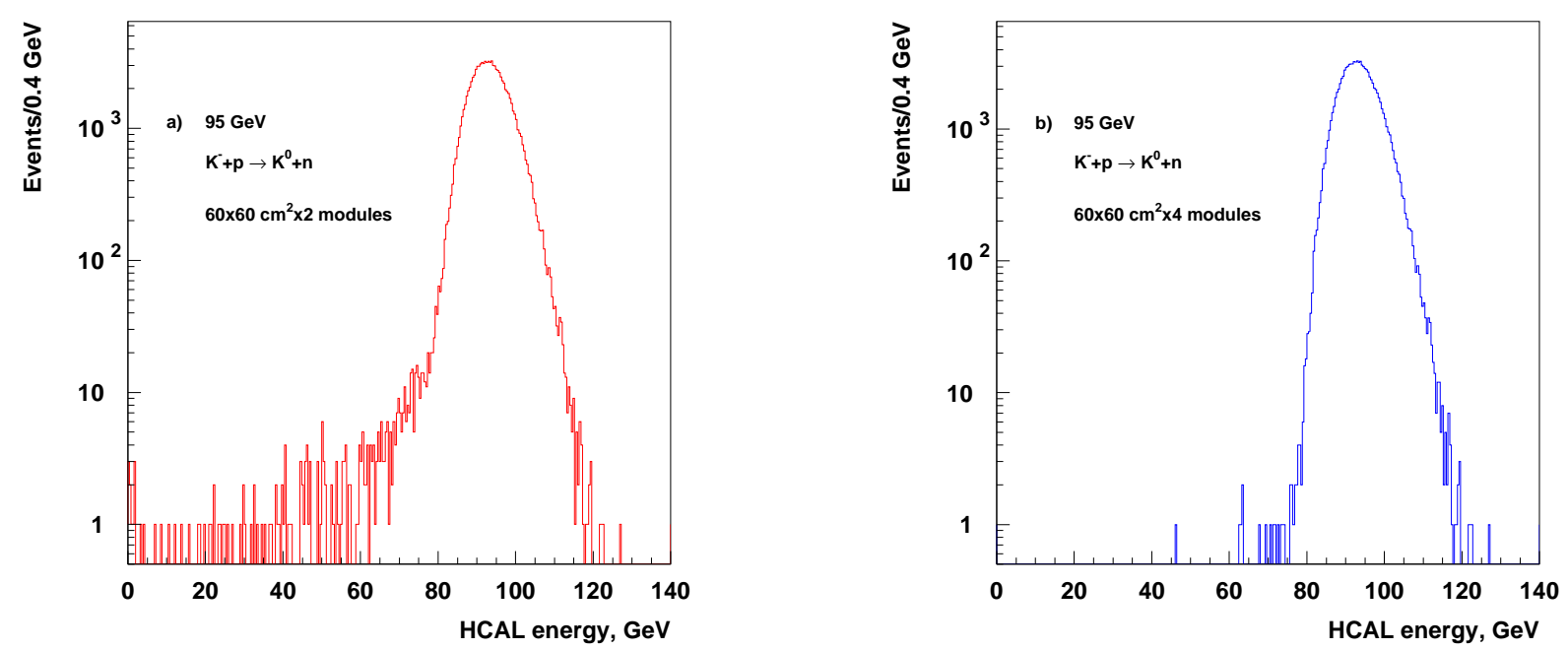

FIG. 3: Expected distributions of energy deposited by $K^{0}$ s with energy $\simeq 95 \mathrm{GeV}$ from the charge exchange reaction Eq. (17) in two (a) and four (b) consecutive HCAL modules. The peak at zero energy in spectrum (a) is due to the punch-through neutral kaons.

ging of the reaction $\pi, K+p \rightarrow M^{0}+n ; M^{0} \rightarrow$ invisible and contribute to background is caused by in-flight $\pi, K \rightarrow \mu, e+\nu$ decays of pions and kaons after they have passed the spectrometer. The background of the low-energy muon admixture in the beam from the $\pi, K \rightarrow \mu \nu$ decays can be due to the following event chain. The decay muon entering the detector decays in flight into a low-energy electron and a neutrino pair, $\mu \rightarrow e \nu \nu$ in the target. The electron then penetrates V1 and V2 without being detected, and deposits all its energy in the HCAL, which is below the threshold $E_{t h} \lesssim 0.5 \mathrm{GeV}$. The probability for this event chain is found to be as small as $P \lesssim 10^{-13}$. Similar background caused by the decays of the beam pions or kaons in the target was also found to be negligible.

More dangerous are the in flight $\pi, K \rightarrow e+\nu$ decays resulting in decay electrons with energy $\lesssim 1 \mathrm{GeV}$, comparable with the energy deposited by the incoming $\pi, K$ in the target. To suppress this background a 2-3 $X_{0}$ preshower detector could be installed upstream of the target. The remaining part of this background is related to the in flight $\pi, K$ decays in the target itself. The combined probability for such decay is suppressed by the small branching fraction of the decays $\operatorname{Br}(\pi(K) \rightarrow e+\nu)<10^{-4}\left(10^{-5}\right)$ down to the level $\lesssim 10^{-9}\left(10^{-10}\right)$. Further suppression of this background could be achieved by using an active target, which is segmented along the beam axis with a separate readout of the signals. An additional suppression factor of 1 order of magnitude is expected form the analysis of the energy deposited in each segment, which should be comparable with the energy deposited by the minimum ionizing particle (MIP). (iv) The fake signature of Eq. (18) could be due to the physical background: a muon scattering on a nucleon, e.g. $\mu^{-} p \rightarrow \nu_{\mu} n$, accompanied by a poorly detected neutron. Taking into account the corresponding cross section and the probability for the recoil neutron to escape detection in the HCAL results in an overall level of this background of $\lesssim 10^{-14}$ per incoming kaon.

In Table \ contributions from the all background processes are summarized for the primary $\pi^{-}$and $K^{-}$beams with energy 40 and $95 \mathrm{GeV}$, respectively. The total background is found (conservatively) to be at the level $\lesssim 1.3 \times 10^{-12}\left(\lesssim 10^{-12}\right)$ per incoming kaon (pion). Therefore, the search accumulated up to $\simeq 10^{12} \pi^{-}$or $K^{-}$ events is expected to be background free. The expected sensitivity in branching fractions is summarized below.

TABLE I: Expected contributions to the total level of background from different background sources estimated per incident $\pi^{-}$and $K^{-}$(see text for details).

\begin{tabular}{lr}
\hline \hline Source of background & Expected level \\
\hline punch-through $K^{0} \mathrm{~s}$, & $\lesssim 10^{-13}$ \\
leading hadron from $\pi$ reactions & $\lesssim 10^{-14}$ \\
low-energy tail of the $\pi^{-}, K^{-}$beam & $\lesssim 10^{-12}$ \\
HCAL nonhermeticity & $\lesssim 10^{-13}$ \\
$\pi^{-}, K^{-} \rightarrow \mu^{-} \nu$ decays in flight & $\lesssim 10^{-13}$ \\
$\pi^{-}\left(K^{-}\right) \rightarrow e^{-} \nu$ decays in flight & $\lesssim 10^{-10}\left(10^{-11}\right)$ \\
$\mu^{-}$induced reactions & $\lesssim 10^{-14}$ \\
\hline Total (conservative) & $\lesssim 10^{-10}$ per $\pi^{-}$ \\
& $\lesssim 10^{-11}$ per $K^{-}$ \\
\hline \hline
\end{tabular}




\section{EXPECTED SENSITIVITY}

To estimate the sensitivity of the proposed experiment a simplified feasibility study based on GEANT4 33. Monte Carlo simulations have been performed for 30-100 $\mathrm{GeV}$ pions and kaons. The ECAL is the hodoscope array of the lead-scintillator counters of the Shashlyk type counters $\left(X_{0} \simeq 2 \mathrm{~cm}\right)$ (see, e.g. Ref.[34]), each with the size of $36 \times 36 \times 400 \mathrm{~mm}^{3}$, allowing for accurate measurements of the lateral energy leak from the target. The target is a block of radiation-hard plastic scintillator with thickness $\simeq 0.5 \lambda_{\text {int }}$ viewed by a photomultiplier. The veto counters are assumed to be $1-2 \mathrm{~cm}$ thick, high-sensitivity LYSO crystal arrays with a high light yield of $\simeq 10^{3}$ photoelectrons per $1 \mathrm{MeV}$ of deposited energy. It is also assumed that the veto's inefficiency for the MIP detection is, conservatively, $\lesssim 10^{-4}$. The hadronic calorimeter is a set of four modules. Each module is a sandwich of alternating layers of iron and scintillator with thicknesses of $25 \mathrm{~mm}$ and $4 \mathrm{~mm}$, respectively, and with a lateral size of $60 \times 60 \mathrm{~cm}^{2}$. Each module consists of 48 such layers and has a total thickness of $\simeq 7 \lambda_{\text {int }}$. The number of photoelectrons produced by a MIP crossing the module is in the range $\simeq 150-200$ ph.e.. In Fig. 2 the distribution of the energy deposited in the HCAL by traversing muons with energy $E_{\mu}=100 \mathrm{GeV}$ is shown. The width of the lhs of the muon peak is defined by the fluctuations of the total number of collected photoelectrons $n_{\text {ph.e. }} \gtrsim 600$ ph.e. with a $\mathrm{rms} \simeq 25$ ph.e.. This should be compared with the effective threshold $\simeq 100$ $\mathrm{MeV}$, or $\simeq 8$ ph.e., for the zero-energy signal which is represented by the distribution of the pedestal sum over the HCAL and also shown for comparison. Thus, one can see that the probability for an event with the MIP energy deposited in the HCAL to mimic the signal due to fluctuations of $n_{p h . e .}$ is negligible.

The hadronic energy resolution of the HCAL calorimeters as a function of the beam energy is taken to be $\frac{\sigma}{E} \simeq \frac{60 \%}{\sqrt{E}}[35]$. The energy threshold for the zero-energy in the HCAL is $0.1 \mathrm{GeV}$. The reported further analysis also takes into account passive materials from the DV vessel walls. To estimate the expected sensitivities we used simulations of the process shown in Fig 1 to calculate fluxes and energy distributions of mesons produced in the target by taking into account the relative normalization of the yield of different meson species $\pi^{0}: \eta: \eta^{\prime}$ and $K^{0}$ from the original publications [36, 37]. The cross section of $\bar{K}^{0}$ production in the reaction (17) can be expressed as [36]

$$
\begin{aligned}
& \frac{\sigma\left(K^{-} p \rightarrow \bar{K}^{0} n\right)}{d t} \simeq(1-G t)\left(\exp \left[c_{\rho} t\right]+R^{2} \exp \left[c_{A} t\right]\right. \\
&-2 R\left[\cos \phi_{+}-G T \cos \phi_{-}\right] \exp \left[\left(c_{\rho}+c_{A}\right) t / 2\right]
\end{aligned}
$$

where $t$ is the four-momentum transfer squared, $G=$ $(33.5 \pm 1.3) \mathrm{GeV}^{-2}, c_{\rho}=(15.5 \pm 0.3) \mathrm{GeV}^{-2}, c_{A}=$ $(8.8 \pm 0.1) \mathrm{GeV}^{-2}, R=0.83 \pm 0.05, \cos \phi_{+}=-0.08 \pm 0.07$, and $\cos \phi_{+}=0.23 \pm 0.02$. This formula gives the para-

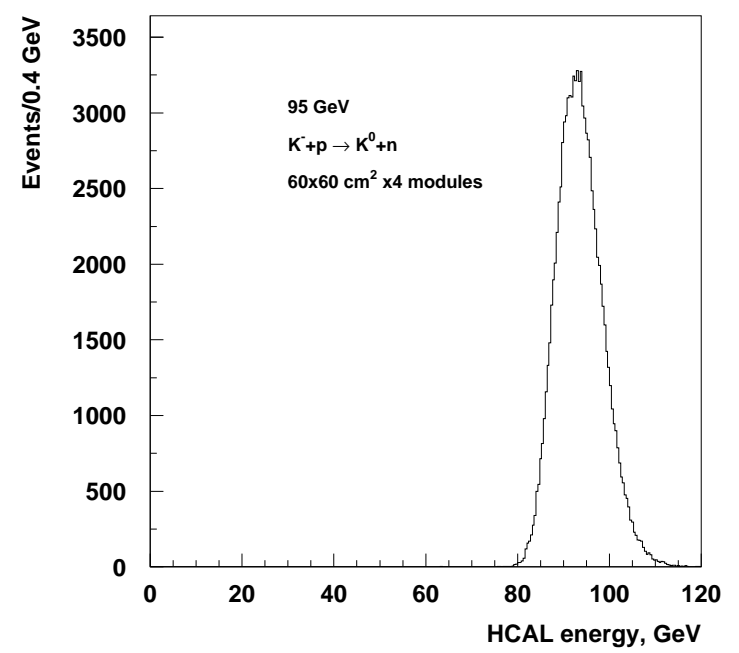

FIG. 4: Expected distribution of the total energy deposited by $K^{0} \mathrm{~s}$ with energy $\simeq 95 \mathrm{GeV}$ from the reaction (17) ) in four HCAL modules.

metric form of the charge-exchange cross sections for the production of neutral kaons over the full phase space, up to $|t| \gtrsim 0.3 \mathrm{GeV}^{-2}$. For $\pi^{0}, \eta, \eta^{\prime}$ we performed similar calculations by using the cross-section parametrization from Ref.[37]. For the purpose of this work, the total $\pi^{0}, \eta, \eta^{\prime}$ and $K^{0}$ production cross sections in the $\pi, K^{-}$chargeexchange reactions in the target were calculated from thier linear extrapolation to the target atomic number. Note, that the yield of $\pi^{0}, \eta, \eta^{\prime}$ and $K^{0}$ is also supposed to be measured in situ (see discussion below). Typically, the branching fractions of the charge-exchange reactions are in the range $\frac{\sigma\left(K^{-} p \rightarrow \bar{K}^{0} n\right)}{\sigma\left(K^{-} p \rightarrow a l l\right)} \simeq \frac{\sigma\left(\pi^{-} p \rightarrow \pi^{0} n\right)}{\sigma\left(\pi^{-} p \rightarrow \text { all }\right)} \simeq 10^{-4}-10^{-3}$ and depend on the beam energy [36, 37].

The calculated fluxes and energy distributions of mesons produced in the target are used to predict the number of signal events in the detector. For a given number of primary kaons $N_{K^{-}}$, the expected total number of $K_{S, L} \rightarrow$ invisible decays occurring within the decay length $L$ of the detector is given by

$$
n_{K}^{i n v}=n_{K_{S}}^{i n v}+n_{K_{L}}^{i n v}
$$

with

$$
\begin{gathered}
n_{K_{S, L}}^{i n v}=k N_{K^{-}} B r\left(K_{S, L} \rightarrow \text { invisible }\right) \\
\cdot \int \frac{\sigma\left(K^{-} p \rightarrow \bar{K}^{0} n\right)}{d t}\left[1-\exp \left(-\frac{L M_{K^{0}}}{P_{K^{0}} \tau_{K_{S, L}}}\right)\right] \zeta \epsilon_{\text {tag }} d t \\
\simeq \zeta \epsilon_{\text {tag }} \operatorname{Br}\left(K_{S, L} \rightarrow \text { invisible }\right) n_{K_{S, L}}^{d e c}
\end{gathered}
$$

where coefficient $k$ is a normalization factor that was tuned to obtain the total cross section of the meson production, $P_{K^{0}}$ and $\tau_{K^{0}}$ are the $K^{0}$ momentum and the lifetime of either $K_{S}$ or $K_{L}$ at rest, respectively, $\zeta$ is the signal reconstruction efficiency, $\epsilon_{\text {tag }}$ is the tagging efficiency of the final state, and $n_{K_{S, L}}^{\text {dec }}$ is the total number of $K_{S, L}$ 


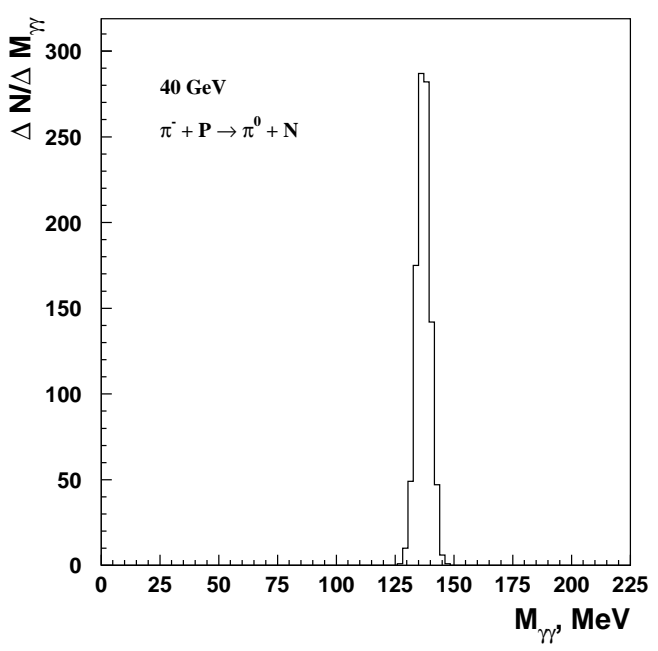

FIG. 5: Expected distribution of the diphoton invariant mass from the reaction (19) in the hodoscopic ECAL, allowing accurate measurements of the photon coordinates, for the incoming pion energy of $40 \mathrm{GeV}$. The spectrum is peaked at the $\pi^{0}$ - mass of $\simeq 135 \mathrm{MeV}$.

decays occurring in the decay volume of length $L$. In this estimate we neglect the $K^{0}$ interactions in the target: the average momentum of the incoming kaons is in the range $<p_{K^{-}}>\simeq 30-100 \mathrm{GeV}$, the decay length $L \simeq 10 \mathrm{~m}$, and the efficiency $\zeta \simeq 0.9$. The tagging efficiency $\epsilon_{\text {tag }}$ is typically $\gtrsim 90 \%$ 36 38]. The contributions from in flight $\pi, K \rightarrow \mu, e+\nu$ decays which could mimic the tagging is negligible. The inefficiency is caused mostly by the inelastic reactions, such as the inelastic charge-exchange reaction $\pi^{-}+p \rightarrow \pi^{0}+N^{* 0}$, with the subsequent isobar decay $N^{* 0} \rightarrow n+\pi^{0}$, or the process $\pi^{-}+p \rightarrow \pi^{0}+\pi^{0}+n$, or the reaction $\pi^{-}+p \rightarrow n^{\prime} \mathrm{s}+X$, etc... All of these reactions are accompanied in the final state by high-energy secondary particles emitted in the forward direction that are subsequently absorbed in the HCAL. Thus, the inefficient tagging of the neutral meson final state would not cause the missing energy background.

In the case of no signal observation, the obtained results can be used to impose upper limits on the previously discussed decays of $\pi^{0}, \eta, \eta^{\prime}, K^{0}$ into invisible final states; by using the relation $n_{K}^{i n v}=n_{K_{S}}^{i n v}+n_{K_{L}}^{i n v}<n_{90 \%}^{i n v}$, where $n_{90 \%}^{i n v}(=2.3$ events $)$ is the $90 \%$ C.L. upper limit for the number of signal events, and Eq. (22), one can determine the expected $90 \%$ C.L. upper limits from the results of the proposed experiment. These bounds - calculated for the total number of $10^{12}$ incident pions or kaons and the background-free case - are summarized in Table II] The limits on $\operatorname{Br}\left(K_{S} \rightarrow\right.$ invisible $)$ and $\operatorname{Br}\left(K_{L} \rightarrow\right.$ invisible $)$ are obtained assuming $n_{K_{L}}^{i n v}=0$ and $n_{K_{S}}^{i n v}$, respectively. Here we also assume that the exposure to the $\pi / K$ beam with the nominal rate is a few months, and that the invisible final states do not decay promptly into the ordinary particles, which would deposit energy in the veto system or HCAL.

As mentioned before, the yield of particles in the reactions (17) and (19) used for normalization and limit calculations is supposed to be determined in separate measurements with the same setup. In Fig. 4 an example of the expected distribution of energy deposited in the HCAL by neutral kaons from the reaction (17) is shown. The distribution is obtained with simplified simulations of $K^{-}$interactions in the target and calculated for a beam energy of $95 \mathrm{GeV}$ and decay length $L \simeq 0.1 \mathrm{~m}$. In this case, the decay length of $K_{S}$ and $K_{L}$ is $\gg L$ and kaons mainly interact in the HCAL before they decay. The candidate events from the primary kaon interaction in the target are selected using the conditions of Eq. (18), but without the requirement of the absence of the energy deposition in the HCAL. One can see that the $K^{0}$ energy spectrum is peaked at maximal beam energy. The distribution is almost background free, has a Gaussian shape and allows one to determine the $K^{0}$ yield with a good accuracy. The values of $n_{K_{S}, L}^{d e c}$ entering Eq.(22) can be easily determined by taking into account that the $K^{0}$ is composed of equal portions of $K_{S}$ and $K_{L}$ of known energies and lifetimes.

The yield of $\pi^{0}, \eta$ and $\eta^{\prime}$ mesons from the $\pi^{-}$chargeexchange reaction in the target can also be determined using similar measurements. In this case, in order to improve the final-state identification, a small hodoscope electromagnetic calorimeter that allows for the reconstruction of two photons from the $\pi^{0}, \eta, \eta^{\prime} \rightarrow \gamma \gamma$ decays can be used. The yield of the $\pi^{0}, \eta$ and $\eta^{\prime}$ mesons can be determined from the low background peaks in the photon pair mass spectrum corresponding to the masses of the decay mesons. In Fig 5 the simulated reconstructed distribution of diphoton invariant mass from the reaction $\pi^{-}+p \rightarrow \pi^{0}+n$ in the hodoscopic Shashlyk ECAL located at a distance $\gtrsim 10 \mathrm{~m}$ from the target is shown for illustration; see, also, e.g., Ref. [38]. Note that (differently from the kaon case) all $\pi^{0}, \eta$ and $\eta^{\prime}$ mesons decay primarily in the target due to their extremely short lifetimes. The measured yield, corrected for the decay photon absorption in the target, directly gives the number $n_{\pi^{0}, \eta, \eta^{\prime}}^{\text {dec }}$ of $\pi^{0}, \eta$ and $\eta^{\prime}$ decays in the target which enter an equation analogous to Eq.(22):

$$
\begin{aligned}
& n_{\pi^{0}, \eta, \eta^{\prime}}^{i n v}=k N_{\pi^{-}} \operatorname{Br}\left(\pi^{0}, \eta, \eta^{\prime} \rightarrow \text { invisible }\right) \\
& \simeq \zeta \epsilon_{\text {tag }} \operatorname{Br}\left(\pi^{0}, \eta, \eta^{\prime} \rightarrow \text { invisible }\right) n_{\pi^{0}, \eta, \eta^{\prime}}^{\text {dec }}
\end{aligned}
$$

The statistical limit on the sensitivity of the proposed experiment is mostly set by the number of accumulated events. However, there is a limitation factor related to the $\mathrm{HCAL}$ signal duration $\left(\tau_{\text {hcal }} \simeq 100 \mathrm{~ns}\right)$ which results in a maximally allowed kaon counting rate $\lesssim 1 / \tau_{H C A L} \simeq 10^{7} \mathrm{~K}^{-} / \mathrm{s}$ above which a significant drop of signal efficiency due to the pileup effect is expected. To evade this limitation, one could implement a special pileup-removal algorithm that allows for a high-efficiency reconstruction of the zero-energy signal properties and shape in high-pileup environments, and then run the ex- 
TABLE II: Expected upper limits on the branching ratios of different decays into invisible final states calculated for the total number of $10^{12}$ incident pions or kaons( see text for details).

\begin{tabular}{lc}
\hline \hline $\begin{array}{l}\text { Expected limits on } \\
\text { the branching ratio }\end{array}$ & Present limit \\
\hline $\mathrm{Br}\left(K_{S} \rightarrow\right.$ invisible $) \lesssim 10^{-8}$ & no \\
\hline $\mathrm{Br}\left(K_{L} \rightarrow\right.$ invisible $) \lesssim 10^{-6}$ & no \\
\hline $\mathrm{Br}\left(\pi^{0} \rightarrow\right.$ invisible $) \lesssim 10^{-8}$ & $<2.7 \times 10^{-7}[2]$ \\
\hline $\mathrm{Br}(\eta \rightarrow$ invisible $) \lesssim 10^{-7}$ & $<1.0 \times 10^{-4}[3]^{a}$ \\
\hline $\mathrm{Br}\left(\eta^{\prime} \rightarrow\right.$ invisible $) \lesssim 10^{-6}$ & $<5.2 \times 10^{-4}[3]^{a}$ \\
\hline \hline
\end{tabular}

${ }^{a}$ These limits are given in Ref. [3] for the values $\frac{\Gamma\left(\eta\left(\eta^{\prime}\right) \rightarrow \text { invisible }\right)}{\Gamma\left(\eta\left(\eta^{\prime}\right) \rightarrow \gamma \gamma\right)}$ and are re-calculated for the ratios $\operatorname{Br}\left(\eta\left(\eta^{\prime}\right) \rightarrow\right.$ invisible $)=$ $\frac{\Gamma\left(\eta\left(\eta^{\prime}\right) \rightarrow \text { invisible }\right)}{\Gamma\left(\eta\left(\eta^{\prime}\right) \rightarrow \text { all }\right)}$, respectively.

periment at the rate $\simeq 1 / \tau_{H C A L} \simeq 10^{7} \mathrm{~K}^{-} / \mathrm{s}$. Thus in the background-free experiment one could potentially expect sensitivities in the $M^{0} \rightarrow$ invisible decay branching ratio that are even higher then those presented in Table II, assuming that the exposure to the high-intensity kaon beam is a few months.

In the case of the $M^{0} \rightarrow$ invisible signal observation, several methods could be used to cross-check the result. For instance, to test whether the signal is due to the HCAL nonhermeticity or not, one could perform measurements with different HCAL thicknesses, i.e. with one, two, three, and four consecutive HCAL modules. In this case the expected background level could be obtained by extrapolating the results to an infinite HCAL thickness. The evaluation of the signal and background could also be obtained from the results of measurements at different beam energies.

An interesting hypothetical question related to the test of the Bell-Steinberger relation is (if the signal is observed) whether it would it be possible in to test the $\mathrm{CP}$ violation in the invisible decays. It it clear that checking it directly (as in the case of the $\mathrm{CP}$ violating decay $K_{L} \rightarrow \pi \pi$ ) would be difficult because the final state is assumed to be unobservable. However, one can perform measurements to see if there is any variation of the zero-energy signal for different lengths of the decay volume. For example, to cross-check whether the signal is mostly from the $K_{S}$ or $K_{L}$ decay, one could remove the decay volume DV and put the HCAL calorimeter behind the veto system. This would not affect the main background sources and still allow for the production of $K_{S} \mathrm{~s}$, but $K_{L}$ decays in front of the HCAL would be suppressed. For measurements with large $L$ in order to ensure that there is no additional background due to the variation of the HCAL hermeticity, e.g. due to the large transverse fluctuation of the hadronic final state, or due to an unexpected (yet unknown) $t$-dependence of the charge-exchange reactions at large $t$ the transverse HCAL size should be large enough. Finally, we note that the presented analysis gives an illustrative order of magnitude for the sensitivity of the proposed experiment and may be strengthened by more detailed simulations of the experimental setup.

\section{CONCLUSION}

Due to their specific properties, neutral kaons are still one of the most interesting probes of physics beyond the standard model from both theoretical and experimental viewpoints. The Bell-Steinberger relation remains the most sensitive probe of CPT invariance in the $K^{0}-\bar{K}^{0}$ system. It connects $\mathrm{CP}$ and $\mathrm{CPT}$ violation in the mass matrix of the kaon system to $\mathrm{CP}$ and $\mathrm{CPT}$ violation in all decay channels of neutral kaons, assuming that there are no significant undiscovered decay modes of either $K_{S}$ or $K_{L}$, such as decays into invisible final states.

In this work we proposed performing an experiment dedicated to the sensitive search for the still unexplored invisible decays of neutral kaons, $K_{S}, K_{L} \rightarrow$ invisible by using available $30-100 \mathrm{GeV}$ kaon beams from the CERN SPS. One of the goals of the proposed search is to clarify the question of how much these decays can influence the Bell-Steinberger analysis of the $K^{0}-\bar{K}^{0}$ system. The experiment is also capable of searching for $\pi^{0}, \eta, \eta^{\prime} \rightarrow$ invisible decays with the SPS pion beams. If the $M^{0} \rightarrow$ invisible decays exist, they could be observed by looking for events with a unique signature: the total disappearance of the beam energy in afully hermetic hadronic calorimeter. A feasibility study of the experimental setup shows that this unique signal of $M^{0} \rightarrow$ invisible decays allows for searches of $K_{S}, K_{L} \rightarrow$ invisible decays with a sensitivity in the branching ratio $\operatorname{Br}\left(K_{S}\left(K_{L}\right) \rightarrow\right.$ invisible $) \lesssim 10^{-8}\left(10^{-6}\right)$, and $\pi^{0}, \eta, \eta^{\prime} \rightarrow$ invisible decays with a sensitivity a few orders of magnitude beyond the present experimental limits. The sensitivitiues in the branching ratios $\operatorname{Br}\left(K_{S}, K_{L} \rightarrow\right.$ invisible $)$ are significantly higher compared to the branching ratios of the $K_{S}, K_{L}$ decay modes which contribute to the present accuracy of the Bell-Steinberger analysis [23].

These results could be obtained with a detector that is optimized for several of properties, namely, i) the intensity and purity of the primary pion and kaon beams, ii) high-efficiency veto counters, and iii) a high level of hermeticity in the hadronic calorimeter are of importance. Large amounts of high-energy hadrons and high background suppression are crucial to improving the sensitivity of the search. To obtain the best limits, a compromise should be found between the background level and the energy and intensity of the beam.

The proposed experiment is complementary to the one recently proposed for a sensitive search for dark photons decaying invisibly to dark-sector particles at the CERN SPS [31, 32]. It also provides interesting motivations for further kaon studies and fits well with the present kaon physics program at CERN (see, e.g., Ref.[39]). 


\section{Acknowledgments}

I would like to thank A. Ceccucci, P. Crivelli, N. Krasnikov, V. Matveev, V. Polyakov, and V. Samoylenko for useful discussions, and A. Dermenev and M. Kir- sanov for their help in calculations. The comments of G.D'Ambrosio and the encouragement of J. Steinberger are very much appreciated.
[1] J. Beringer et al. (Particle Data Group), Phys. Rev. D 86, 010001 (2012).

[2] A. V. Artamonov et al. (E949 Collaboration), Phys. Rev. D 72, 091102 (2005).

[3] M. Ablikim et al. (BES Collaboration), Phys. Rev. 87, 012009 (2013).

[4] C.L. Hsu et al. (Belle Collaboration), Phys. Rev. D 86, 032002 (2012).

[5] B. Aubert et al., (BABAR Collaboration), Phys. Rev. Lett. 103, 251801 (2009).

[6] M. Ablikim et al. (BES Collaboration), Phys. Rev. Lett. 100, 192001 (2008).

[7] P. Rubin et al., (CLEO Collaboration), Phys. Rev. D 75, 031104 (2007).

[8] S.N. Ahmed et al., (SNO Collaboration), Phys. Rev. Lett. 92, 102004 (2004).

[9] H.O. Back et al., (Borexino Collaboration), Phys. Lett. B 563, 23 (2003).

[10] T. Araki et al., Phys. Rev. Lett. 96, 101802 (2006).

[11] V.I. Tretyak, V.Yu. Denisov, and Yu.G. Zdesenko, JETP Lett. 79, 106 (2004), Pisma Zh. Eksp. Teor. Fiz. 79, 136 (2004); nucl-ex/0401022.

[12] H.V. Klapdor-Kleingrothaus, I.V. Krivosheina, and I.V. Titkova, Phys. Lett. B 644, 109 (2007).

[13] G.Banet al., Phys. Rev. Lett. 99, 161603 (2007).

[14] A.P. Serebrov et al., Phys. Lett. B 663, 181 (2008).

[15] M. Sarrazin, G. Pignol, F.Petit, V.V. Nesvizhevsky, Phys. Lett. B 712, 213 (2012).

[16] S.N. Gninenko, N.V. Krasnikov, and A. Rubbia, Phys. Rev. D 67, 075012 (2003).

[17] A. Badertscher, P. Crivelli, U. Gendotti, S.N. Gninenko, V .Postoev, A. Rubbia, V. Samoylenko and D. Sillou, Phys. Rev. D 75, 032004 (2007)

[18] S.N. Gninenko, N.V. Krasnikov, and V.A. Matveev, Phys. Rev. D 87, 015016 (2013); arXiv:1209.0060 [hepph].

[19] S.N. Gninenko, Phys. Rev. D 76, 055004 (2007).

[20] P. Crivelli, A. Belov, U. Gendotti, S. Gninenko, and A. Rubbia, JINST 5, P08001 (2010); arXiv:1005.4802 [hepex].

[21] J. S. Bell and J. Steinberger, In Oxford International Symposium Conference on Elementary Particles, Oxfors, England, September 19-25, 1965. edited by L. Wolfenstein (Rutherford High Energy Laboratory, Chilton, 1965), p.42..
[22] J. Steinberger, " $K^{0}$ Decay and CP Violation", CERN 70-1, (1970).

[23] M. Antonelli and G. D'Ambrosio, "CPT Invariance Tests in Neutral Kaon Decay" p.839, in [1].

[24] L. Maiani, in The Second DAPHNE Physics Handbook. Two Volumes, edited by L. Maiani, G. Pancheri, and N. Paver, INFN 1202 (1995), (Frascati, 1995).

[25] G. D?Ambrosio, G. Isidori, and A. Pugliese, in The Second DAPHNE Physics Handbook. Two Volumes, edited by L. 739 Maiani, G. Pancheri, and N. Paver, INFN 1202 (1995), (Frascati, 1995).

[26] P. Bloch and L. Tauscher, Annu. Rev. Nucl. Part. Sci. 53, 123 (2003).

[27] F. Ambrosino et al., [KLOE Collab.], J.High Energy Phys. 0612, 011 (2006) arXiv:hep-ex/0610034.

[28] A. Angelopoulosetal. et al., CPLEAR Collaboration, Phys. Lett. B471, 332 (1999).

[29] K. R. Schubert, L. Li Gioi, A. J. Bevan, A. Di Domenico, arXiv:1401.6938 [hep-ex].

[30] See, for example, http://sba.web.cern.ch/sba/

[31] S.N. Gninenko, Phys. Rev. D 89, 075008 (2014); arXiv:1308.6521 [hep-ph].

[32] S. Andreas et al., arXiv:1312.3309 [hep-ex]; CERNSPSC-2013-034 / SPSC-P-348.

[33] S. Agostinelli et al. (GEANT4 Collaboration), Nucl. Instrum. Methods Phys. Res., Sect. A 506, 250 (2003); J. Allison et al. (GEANT4 Collaboration) IEEE Trans. Nucl. Sc. 53, 270 (2006).

[34] G.S. Atoian, V.A. Gladyshev, S.N. Gninenko, V.V. Isakov, A.V. Kovzelev, E.A. Monich, A.A. Poblaguev, A.L. Proskuryakov, I.N. Semenyuk, V.G. Lapshin et al., Nucl. Instrum. Methods Phys. Res., Sect. A 320, 144 (1992).

[35] G.A. Alekseev et al., Nucl. Instrum. Meth. A 461, 381 (2001).

[36] F. Binon et al., Il Nuovo Cimento 64 A, 89 (1981).

[37] V.N. Bolotov et al., Nucl. Phys. B 85, 158 (1975).

[38] F. Binon et al., Z. Phys. C 9, 109 (1981).

[39] A. Ceccucci, "The Kaon Physics Programme at CERN" Proceedings, 5th International Seminar on High Energy Physics (Quarks 2008) 23-29 May 2008. Sergiev Posad, Russia http://quarks.inr.ac.ru/2008/proceedings/p4

[40] S.N. Gninenko, N.V. Krasnikov. Paper in preparation 